\title{
A simple snowpack/cloud reflectance and transmittance model from microwave to ultraviolet: the ice-lamella pack
}

\author{
Christian Mätzler \\ Department of Microwave Physics, Institute of Applied Physics, University of Bern, Sidlerstrasse 5, CH-3012 Bern, Switzerland
}

\begin{abstract}
Modeling the reflectance and transmittance of strong volume scatterers is a delicate task. Slightly different approaches can lead to different results, making comparisons difficult. Here a simple, analytic multiple-scattering model is presented as a possible reference for comparisons and also for better understanding of the physics involved. The model quantifies the transmittance and reflectance of homogeneously distributed scatterers within slabs of any thickness. The simplicity of the model is given by the onedimensional geometry, a system consisting of freely arranged ice lamellae in air. Although direct application of the model will be limited, it gives a spectral description of ice clouds and snow packs over a very broad spectral range from microwave to ultraviolet. As well as the transmittance and reflectance, the model gives the emittance through Kirchhoff's law. Comparison with other models shows, on the one hand, agreement with current snow models in the spectral description, and on the other, some quantitative inconsistencies between all of them. It appears that the lamella pack produces the same optical spectra as an average snow model, with spherical ice grains whose radius corresponds to about the lamella thickness, whereas microwave spectra appear to be slightly different.
\end{abstract}

\section{INTRODUGTION}

The purpose of optical models of snow and clouds is to quantify the measurable reflectance and transmittance as functions of wavelength and medium properties, especially particle size. The "optical" grain-size derived from measured optical data is the direct inversion of such models. If different models give different results we have the problem of ambiguity. The present paper shows that this problem exists, and proposes a way to solve it. Modeling the reflectance and transmittance of strongly scattering media is a delicate task. Different approaches using slightly different assumptions, even within the same model, lead to different results, making comparisons difficult. A major problem arises from the fact that the shape and size of scattering particles are often not known, and even if they are known cannot easily be described. As a result, a certain degree of arbitrariness in the choice of these quantities evolves in the different models. It would be highly desirable to have a simple, analytic multiple-scattering model available as a standard for comparison and to better understand the physics involved. Such a model can give detailed insight into the spectral behavior of scattering and emitting media, even if not all the properties are realistic. The ice-lamella system presented here represents such a model. It is purely one-dimensional, with all lamellae being parallel, all waves being plane waves and all wave interactions being limited to two directions (incident and reflected waves at the same linear polarization).

In contrast to the optical industry where controlled glass-lamellae systems play important roles in adjusting light and heat transmittance and reflectance, in the present case, the lamellae are assumed to be freely arranged and slightly variable in thickness, leading to a kind of average behavior. Free arrangement means that the expected icevolume fraction $v$ is independent of position, which also means that a fraction $v$ of particle surfaces are in contact. This property assures, on average, incoherent interactions between different lamellae even at high values of $v$, a rather surprising result found by Mätzler (1997, 1998a). The model gives a surprisingly realistic spectral description of ice clouds and snowpacks from microwave to the ultraviolet (UV) of homogeneous situations. As well as the transmittance and reflectance, the model gives the emittance through Kirchhoff's law. The model is described for vertical incidence in section 2, and the extension to oblique incidence is straightforward. In section 3 the model results are presented, and comparisons are made with other snow-reflectance models, the emphasis being placed on comparison of effective grain-sizes and on the spectral behavior.

\section{THE MODEL}

\subsection{Model geometry}

Let us assume a model for a purely one-dimensional scattering medium to represent either a snowpack or an ice cloud, i.e. a pack of thin, horizontal ice sheets of mean thickness $d$, packed to a volume fraction $v(0<v<1)$, so that on average there are $N$ lamellae per meter depth (Fig. 1). The lamellae are assumed to be freely arranged, a concept introduced by Mätzler (1997).

Expressing $N$ by $v$ and $d$ we obtain

$$
N=v / d
$$

The main advantage of this geometry is that the reflectance can be computed simply and exactly, in the sense that only two spatial directions are involved in the scattering process, a forward and a backward ray. In this case the integro-differential radiative transfer equation decays into a pair of 


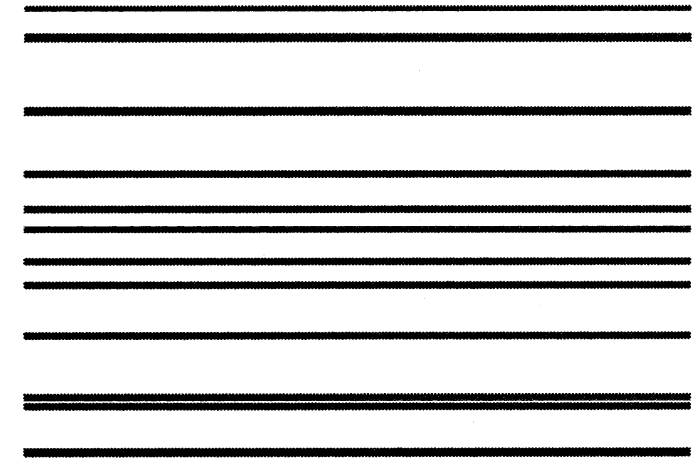

Fig. 1. Pack of freely arranged ice lamellae, representing the ice-lamella system, a model of a cloud or of a snowpack. Freely arranged lamellae can be in direct contact (second from bottom), thus increasing the average thickness.

first-order differential equations (see Equation (6)) identical to the ones used in the two-flux model for which exact solutions are known. Furthermore, certain icy scatterers are indeed horizontally aligned (ice lenses, horizontally aligned snow layers, plate-like ice crystals in cirrus clouds in nonturbulent condition), and thus are favorably approximated. Since volume scattering of spherical or other ice grains is also well approximated by two-stream theories (Meador and Weaver, 1980), their results can be related and compared to the present simple model.

\subsection{Reflection of a single ice lamella}

For a dielectric lamella with negligible losses, the reflectivity is given by the Airy equation(Born and Wolf, 1980)

$$
r=2 r_{1} \frac{1-\cos (2 P)}{1+r_{1}^{2}-2 r_{1} \cos (2 P)},
$$

where $r_{1}$ is the Fresnel reflectivity of the air-ice interface and $P$ is the one-way phase through the lamella. For vertical incidence these quantities are given by

$$
r_{1}=\left|\frac{n-1}{n+1}\right|^{2}
$$

and

$$
P=n^{\prime} k d,
$$

where $n=n^{\prime}+i \cdot n^{\prime \prime}$ is the complex refractive index of ice and $k$ is the vacuum wavenumber.

\subsection{Average reflectivity of a lamella}

Since $d$ is assumed to be slightly different for different lamellae, the phase terms in Equation (2) are smeared out when averaged over many lamellae, except for very small values of $P$. For the same reason, coherent superpositions of reflections at different lamellae disappear due to the variable distance between them, i.e. the free arrangement of the lamellae. Noting that $r_{1} \ll 1$, the denominator of Equation (2) can be approximated by 1 . The average lamella reflectivity $r_{\mathrm{av}}$ can then be written as

$$
r_{\mathrm{av}}= \begin{cases}4 r_{1} \sin ^{2} P ; & \mathrm{P}<3 \pi / 4=2.356 \\ 2 r_{1} ; & \mathrm{P} \geq 2.356 .\end{cases}
$$

For a small phase, Equation (5) gives the coherent reflectivity of the lamella through the first maximum of the sine function (at one-quarter wavelength), and it provides a continuous transition from the coherent to the incoherent situation at larger thickness (or larger phase).

\subsection{Two-flux scattering coefficient}

Scattering consists of reflections of incident radiation at the ice lamellae. Since all lamellae are horizontally aligned, the reflections from vertically incident radiation result in radiation propagating in the opposite direction. Freely arranged scatterers interact incoherently (Mätzler, 1998a), so a radiative-transfer treatment can be used to describe the scattering of the total layer. In this one-dimensional geometry, the so-called two-flux or two-stream model (e.g. Ishimaru, 1978 ) is exact. In this model, the radiative transfer of the up- and downwelling intensities, $I_{1}(z)$ and $I_{2}(z)$, respectively, can be described by the following pair of equations (emission being omitted here, but included later), assuming an upward-directed $z$ axis:

$$
\frac{\mathrm{d} I_{1,2}}{d z}= \pm \gamma_{\mathrm{a}} I_{1,2}+\gamma_{\mathrm{s}}\left(I_{2}-I_{1}\right)
$$

where the minus sign in the first term on the right applies for $I_{1}$ and the plus sign for $I_{2}$. For constant coefficients these equations are solved analytically, leading to the results given below. For $N$ lamellae per meter depth the scattering coefficient $\gamma_{\mathrm{s}}$ is given by $\gamma_{\mathrm{s}}=N r_{\mathrm{av}}$. However, due to the free arrangement, this quantity is reduced by the probability $(1-v)$ of two adjacent lamellae being in contact, so $\gamma_{\mathrm{s}}$ is given by

$$
\begin{aligned}
\gamma_{\mathrm{s}} & =N(1-v) r_{\mathrm{av}} \\
= & \left\{\begin{array}{cl}
\frac{4 v(1-v) r_{1} \sin ^{2} P}{d} ; & P=n^{\prime} k d<3 \pi / 4=2.356 \\
\frac{2 v(1-v) r_{1}}{d} ; & P \geq 2.356
\end{array}\right.
\end{aligned}
$$

The contacting lamellae have an increased total thickness, so we can introduce the average thickness as $d_{\mathrm{av}}=d /(1-v)$. This quantity probably represents a measured mean thickness more closely than $d$. Thus Equation (7) can also be expressed by

$\gamma_{\mathrm{s}}=\left\{\begin{array}{cl}\frac{4 v r_{1} \sin ^{2} P}{d_{\mathrm{av}}} ; & P=n^{\prime} k d_{\mathrm{av}}(1-v)<3 \pi / 4=2.356 \\ \frac{2 v r_{1}}{d_{\mathrm{av}}} ; & P \geq 2.356 . .^{*}\end{array}\right.$

\subsection{Two-flux absorption coefficient}

So far, dielectric losses have been ignored. Small losses can be included by the absorption coefficients of ice $\gamma_{\mathrm{a} \text {,ice }}$ and air $\gamma_{\mathrm{a}, \text { air }}$. The resulting absorption coefficient $\gamma_{\mathrm{a}}$ of the lamella model is

$$
\gamma_{\mathrm{a}}=v \gamma_{\mathrm{a}, \mathrm{ice}}+(1-v) \gamma_{\mathrm{a}, \mathrm{air}} .
$$

In the case of high dielectric losses, absorption happens locally within the topmost lamella, leading to Equation $\left(9^{\prime}\right)$ below.

* $d$ is the correlation length of the one-dimensional medium. With constant $d$ and increasing $v$, more and more lamellae touch an adjacent one until $v$ approaches 1 when the medium consists of thick ice plates separated by a few thin air gaps of thickness $d$. Thus the average ice-lamella thickness depends on density, whereas the basic structure parameter does not. See Equations (19-21) for other geometrical considerations. 


\subsection{Reflectance, transmittance and emittance of the slab}

Let us neglect atmospheric absorption, and let us assume that the slab of height $h$ (thus consisting of $N \cdot h$ lamellae) is situated above a non-reflecting background. Then the reflectivity (or reflectance), $r$, and the transmissivity (transmittance), $t$, of the slab are determined by the two-flux model, defining the reflectivity $r_{0}$ for infinite thickness, the transmission function $t_{0}$ and the damping coefficient (eigenvalue) $\gamma_{2}$ of the model (e.g. Mätzler, 1987):

$$
\begin{aligned}
& r=r_{0} \frac{1-t_{0}^{2}}{1-r_{0}^{2} t_{0}^{2}} ; \quad \text { and } \quad t=t_{0} \frac{1-r_{0}^{2}}{1-r_{0}^{2} t_{0}^{2}} ; \\
& \gamma_{\text {a,ice }} d<1
\end{aligned}
$$

where the model parameters are given by

$$
t_{0}=\exp \left(-\gamma_{2} h\right) ; r_{0}=\frac{\gamma_{\mathrm{s}}}{\gamma_{\mathrm{s}}+\gamma_{\mathrm{a}}+\gamma_{2}} ; \gamma_{2}=\sqrt{\gamma_{\mathrm{a}}^{2}+2 \gamma_{\mathrm{a}} \gamma_{\mathrm{s}}}
$$

An exception to Equation (9) must be made when the ice lamella becomes opaque; then only the reflections at lamellae near the top of the pack will contribute. A useful approximation for the pack reflectivity and transmissivity is obtained by including incoherent reflections at the three topmost air-ice interfaces and neglecting multiple scattering:

$$
r=r_{1}\left[1+2 \exp \left(-2 \gamma_{\mathrm{a}, \text { ice }} d\right)\right] ; \quad t=0 ; \quad \gamma_{\mathrm{a}, \text { ice }} d>1 .
$$

Finally, the emissivity (or emittance) $e$ of the slab is obtained from Kirchhoff's law, stating that $e$ is equal to the absorptivity $a$ :

$$
a=e=1-t-r \text {. }
$$

This equation follows from energy conservation. The emissivity of the whole (slab and background) system is given by $e_{0}=1-r=e+t$.

\section{DISGUSSION}

For thin packs, i.e. small $h\left(\ll 1 / \gamma_{\mathrm{s}}\right)$, the slab reflectivity simplifies to $r \cong \gamma_{\mathrm{s}} h$. On the other hand, for a sufficiently deep pack, the reflectivity is only a function of the ratio $x=\gamma_{\mathrm{s}} / \gamma_{\mathrm{a}}$ :

$$
\begin{gathered}
r=r_{0}=\frac{x}{x+1+\sqrt{1+2 x}} \\
r_{0} \cong 1-\sqrt{\frac{2}{x}} .
\end{gathered}
$$

The often used Approximation (13) is approached for large values of $x$ (e.g. for snow at visible wavelengths). Inserting $x$ from Equations $\left(7^{\prime}\right)$ and (8) for $\gamma_{\mathrm{a}, \text { air }}=0$ and for large $P$ we obtain

$$
x=\frac{2 r_{1}}{\gamma_{\mathrm{a}, \text { ice }} d_{\mathrm{av}}},
$$

and thus from Approximation (13) we find

$$
r_{0} \cong 1-K \sqrt{\gamma_{\mathrm{a}, \text { ice }} d_{\mathrm{av}}}
$$

a well-known approximation for the reflectance of a strongly volume-scattering object (e.g. Bohren, 1987). Here, the constant $K$ is given by $r_{1}^{-1 / 2}$; thus for lossless ice we obtain from Equation (3):

$$
K=\frac{n^{\prime}+1}{n^{\prime}-1} .
$$

Inserting the refractive index of ice in the visible range $\left(n^{\prime}=1.33\right)$, we obtain $r_{1}=0.020$ and $K=7.06$. According to an early snowpack model of spherical ice particles (Bohren and Barkstrom, 1974), the reflectance of a deep snowpack can be written as

$$
r_{0} \cong 1-5.96 \sqrt{\gamma_{\mathrm{a}, \text { ice }} D_{\mathrm{BB}}}
$$

where $D_{\mathrm{BB}}$ is the sphere diameter of the Bohren and Barkstrom model. This result agrees with Approximation (15) by choosing

$$
d_{\mathrm{av}}=0.61 D_{\mathrm{BB}} .
$$

Another comparison can be made using the snow model of Wiscombe and Warren (1980). Reflectivities of thick snowpacks computed with this model for different grain diameters $D_{\text {WW }}$ were taken from Marshall (1989) and from Sergent and others (1993) at a wavelength of $1 \mu \mathrm{m}$, and the results are shown by the data on the upper curve of Figure 2. The curve represents Equation (12) for $x=4.915 \mathrm{~mm} / D_{\mathrm{Ww}}$. Comparing this result with Equation (14) for $r_{1}=0.0171\left(n^{\prime}=1.301\right)$ and $\gamma_{\mathrm{a}, \text { ice }}=0.024 \mathrm{~mm}^{-1}$, we find $x=1.42 / d_{\mathrm{av}}$, so agreement is achieved with the lamella model if

$$
d_{\mathrm{av}}=0.29 D_{\mathrm{WW}} \cdot
$$

The lower curve in Figure 2 represents the model of De Haan and others (1987), with $x=2.69 \mathrm{~mm} / D_{\mathrm{DH}}$. Agreement with the lamella model is achieved if the De Haan grain diameter $D_{\mathrm{DH}}$ is given by

$$
d_{\mathrm{av}}=0.53 D_{\mathrm{DH}} \text {. }
$$

Comparison of Equations $(18 \mathrm{a}-\mathrm{c})$ indicates that different snow-reflectance models lead to slightly different results (all within about a factor of 2) with respect to the grain diameter. This is why a reference standard could help to identify differences between models and ultimately improve the modeling work. The above comparison also means that the present simple model fits well within the snow-reflectance models. On average, the lamella thickness is about equal to the grain radius.

Another comparison of the lamella-pack model was made with data of the NASA Adavanced Spaceborne Thermal Emission and Reflection Radiometer (NASA-ASTER spectral library (http://asterweb.jpl.nasa.gov). The results are

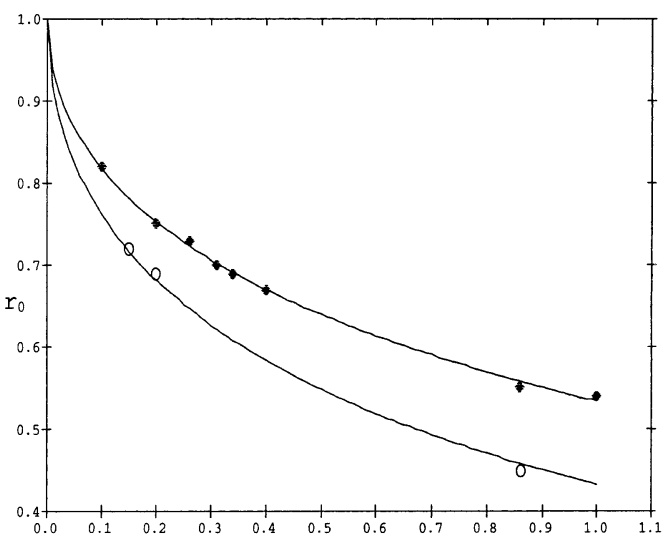

$$
\text { arain diameter in } \mathrm{nm}
$$

Fig. 2. Decrease of the reflectance $r_{0}$ of pure snow (wavelength $1 \mu \mathrm{m}$ ) with increasing grain diameter $D$ of spherical ice grains. Data points (diamonds) along the upper curve are computed using the model of Wiscombe and Warren (1980); the curve represents Equation (13) with $x=4.915 \mathrm{~mm} / D$. The lower curve represents Equation (13) with $x=$ $2.69 \mathrm{~mm} / D$. The data points were computed by Sergent and others (1998), using the model of De Haan and others (1987). 
shown in Figure 3 for three different effective grain-sizes $D_{\mathrm{AS}}=0.024,0.082$ and $0.178 \mathrm{~mm}$. The lamella thickness, $d$, was assumed to be equal to $D_{\mathrm{AS}}$, and a constant ice-volume fraction $v=0.1$ was assumed. The spectral ice data were taken from Warren (1984). According to the description of the ASTER library, the ASTER spectra were modeled based on broad-band measurements $(2-14 \mu \mathrm{m})$ made by Salisbury and others (1994) at the Johns Hopkins University Infrared Laboratory, Baltimore, MD. The model quoted is Wald's (1994). The agreement between the spectra of the lamella pack and the ASTER snowpack is excellent. Especially at wavelengths of $<1.4 \mu \mathrm{m}$, the ASTER data and the lamella-pack model give almost identical results. From this coincidence it can be concluded that the ASTER grain-size corresponds to the original lamella thickness $d$ for $v=0.1$. Expressing the comparison by $d_{\text {av }}$ we have

$$
d_{\mathrm{av}}=1.1 D_{\mathrm{AS}} .
$$

Unfortunately, in the ASTER library the "grain-size" $D_{\mathrm{AS}}$ is not clearly defined. After discussion with the authors of the database it seems probable that "ASTER grain-size" means radius. In this case the agreement with the results of Equations $(18 \mathrm{a}-\mathrm{c})$ and Figure 2 is much better. In fact, Wald (1994) used "radius" and "grain-size" as synonyms.

From updated spectral information on the complex refractive index of pure-water ice (Warren, 1984; Mätzler, 1998b), microwave to UV reflectivity and transmissivity spectra were computed for given packs. Examples of reflectance and transmittance data of two $10 \mathrm{~cm}$ thick snowpacks at a temperature of $266 \mathrm{~K}$ are shown in Figure $4 \mathrm{a}$ and b. Figure $4 \mathrm{c}$ shows the spectra of an ice cloud (shortest wavelength is $200 \mathrm{~nm}$ ). The corresponding reflectivity spectra for infinite thickness are also shown. The computations are based on Equations (9), $\left(9^{\prime}\right)$ and (10). At lower frequencies where the phase $P$ is small, we obtain a reflectivity which increases with increasing $k^{2} d$, i.e. with frequency, so the emissivity decreases, as is observed for dry snow in the microwave range. In Figure $4 \mathrm{a}$ and $\mathrm{b}$ the reflectivity in the $2-100 \mathrm{GHz}$ range is compared with the results of the recent Microwave Emission Model of Layered Snowpacks (MEMLS; Wiesmann and others, 1998, Wiesmann and Mätzler, 1999;) for the same thickness, density and temperature, and for correlation lengths $p_{\text {MEMLS }}$ of the isotropic heterogeneity fitted to the

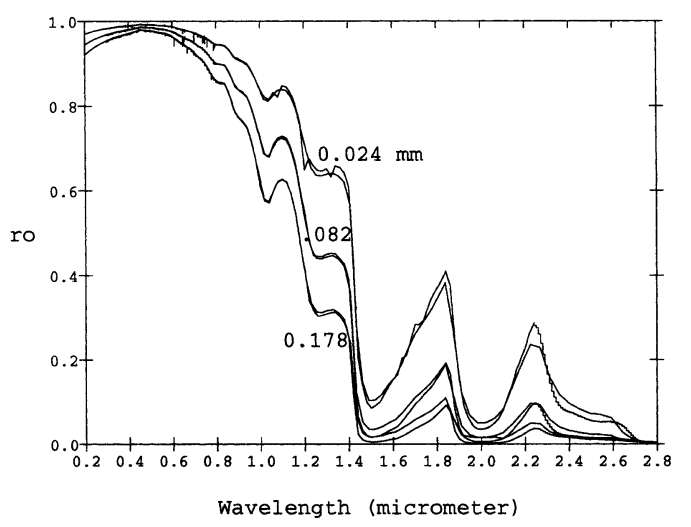

Fig. 3. Reflectance of thick snowpacks vs wavelength between 0.2 and $2.8 \mu \mathrm{m}$ for three effective grain-sizes: $0.024 \mathrm{~mm}$ (uppermost pair of curves), $0.082 \mathrm{~mm}$ (middle pair) and $0.178 \mathrm{~mm}$ (lowest pair). The smoother curves represent the lamella-pack model with the lamella thickness equal to the grain-size for $v=0.1$; the noisier curves represent data from the ASTER spectral library. present data. It is found that $p_{\text {MEMLS }}$ is significantly larger than $d$, and its influence on the scattering coefficient is stronger than for $d$. Indeed, three-dimensional Rayleigh scattering increases with $k^{4}\left(p_{\text {MEMLS }}\right)^{3}$, whereas in the one-dimensional geometry, scattering increases with $k^{2} d$. The shape of the MEMLS spectra (Fig. 4a and b) is also slightly steeper than in the present model. Thus there is a functional difference between scattering in one- and three-dimensional heterogeneity at microwave frequencies, whereas at optical frequencies both types of heterogeneity produce coincident spectra and coincident grain-size dependence.

A comparison between the two correlation lengths $d$ and $p_{\text {MEMLS }}$ follows from geometrical considerations, referring to the specific surface $s=S / V$ of a granular medium where $S$ is the total surface of particles within volume $V$. In the three-dimensional case, the equation of Debye and others (1957) applies:

$$
s=4 v(1-v) / p_{\mathrm{MEMLS}},
$$
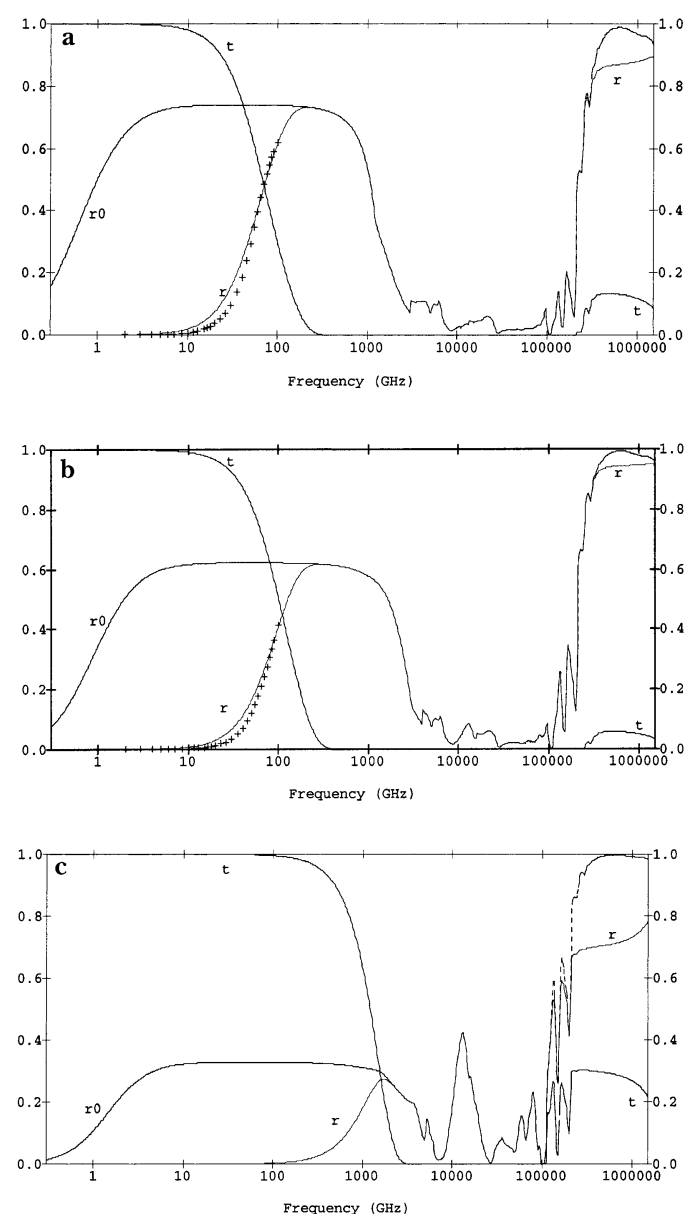

Fig. 4. ( $a, b)$ Radio to $U V$ spectra of transmissivity $t$ and reflectivity $r$ of a thin snowpack consisting of a $10 \mathrm{~cm}$ deep ice-lamella pack with $d=0.05 \mathrm{~mm}, v=0.1$ (a) and $d=0.02 \mathrm{~mm}, v=0.1(b)$. Also shown is the reflectivity $r_{0}$ of the same snow, but at infinite thickness. The data points labeled with crosses are MEMLS results of $r$ for the same snow density, thickness and temperature $(266 K)$, but with $p_{\mathrm{MEMLS}}=0.2 \mathrm{~mm}$ (a) and $p_{\mathrm{MEMLS}}=0.12 \mathrm{~mm}$ (b). (c) Radio to $U V$ spectra of transmissivity $t$ and reflectivity $r$ of an ice cloud consisting of a $100 \mathrm{~m}$ deep ice-lamella pack with $d=3 \mu \mathrm{m}, v=2 \times 10^{-6}$. Also shown is the reflectivity $r_{0}$ of the same cloud, but at infinite thickness. Absorption in moist air was neglected. 
whereas in the one-dimensional case of Figure 1, $s$ is given by

$$
s=2 v(1-v) / d .
$$

Comparing Equations (19) and (20) gives

$$
p_{\text {MEMLS }}=2 d \text {. }
$$

In view of Equation (21) the discrepancy in Figure $4 \mathrm{a}$ and $\mathrm{b}$ between $p_{\text {MEMLS }}$ and $d$ is not too severe.

For the general behavior of the model as seen in Figure $4 \mathrm{a}-\mathrm{c}$, the following can be noted: There is a broad maximum of the infinite reflectivity $r_{0}$ in the $10-1000 \mathrm{GHz}$ range. This maximum decreases with decreasing $d$, whereas the maxima increase at short wavelengths. This property is intrinsic to volume scattering when the wavelength changes from larger to smaller than the characteristic size of the scatterers. The reason why the maximum of $r_{0}$ is so flat over the $10-1000 \mathrm{GHz}$ range is the common behavior of $\gamma_{\mathrm{a}}$ and $\gamma_{\mathrm{s}}$ in this frequency range, both increasing with the square of frequency. The behavior is different at $<10 \mathrm{GHz}$ where $\gamma_{\mathrm{a}}$ converges to a frequency-independent value, leading to an increase of $r_{0}$ with frequency squared.

The transmissivity shows a high-frequency cut-off near $100 \mathrm{GHz}$ for the $10 \mathrm{~cm}$ snowpacks and near $1000 \mathrm{GHz}$ for the ice cloud. The difference is mainly due to the different water-equivalent depth, decreasing from $10 \mathrm{~mm}$ for the snowpack to $0.2 \mathrm{~mm}$ for the cloud. The transition from Equation (9) to Equation (9'), from transparent to opaque lamellae, takes place deep within the cut-off region, at frequencies of $>4000 \mathrm{GHz}$, with a return to Equation (9) at a wavelength of $<3 \mu \mathrm{m}\left(f>10^{5} \mathrm{GHz}\right)$. At the transition point, the reflectivity for Equation $\left(9^{\prime}\right)$ is larger than for Equation (9), leading to visible jumps in the spectra. The transition from incoherent to coherent lamella reflections, as expressed by $P=3 \pi / 4$ in Equation (7), occurs in the decreasing part of $r$, near $1000 \mathrm{GHz}$ in Figure 4a and near $3000 \mathrm{GHz}$ in Figure 4b (see the slight change in slope).

\section{GONGLUSIONS}

A simple, physical, multiple-scattering model was presented for describing the reflectance and the transmittance (Equations (9) and (10)) over a very large frequency range in a volume-scattering medium, such as snowpacks and clouds, consisting of ice and air. The one-dimensional geometry consists of a slab of freely arranged, horizontally aligned ice lamellae of a given original thickness $d$. Due to occasional contacts between adjacent lamellae, the average lamella thickness $d_{\mathrm{av}}$ is slightly larger than $d$. Either one of these parameters describes the structure, together with the ice-volume fraction $v$. The shortwave reflectance spectra up to a wavelength of $2.8 \mu \mathrm{m}$ coincide with snow spectra modeled for spherical ice grains using Mie theory for grain radii about equal to $d_{\mathrm{av}}$. The decrease of the reflectance with increasing grain-size is the same in both types of model. A certain discrepancy between different snow-reflectance models was observed (Equations 18a-d).

Concerning the microwave range, there is no general agreement between the ice-lamella model and scattering in a three-dimensional heterogeneity. Nevertheless the present one-dimensional geometry gives an approximate agreement with reflectivities computed with a snow-emission model if the correlation length $p_{\mathrm{MEMLS}}$ of the three-dimensional medium is properly adjusted to $d$. By using the information available on the complex refractive index of ice, very broad-band spectra for snowpacks and clouds can be constructed from the formulae presented here. Due to the simplicity of the ice-lamella pack, this model could be used as a reference in the development, validation and improvement of more elaborate models.

\section{ACKNOWLEDGEMENTS}

The author would like to thank T. Grenfell and S. Warren for providing the ice refractive-index data from Warren (1984) and for an updated version. Thanks also go to the Jet Propulsion Laboratory, California Institute of Technology, for providing the ASTER data and to A. Wald and J. Salisbury for detailed discussion of aspects of their papers.

\section{REFERENGES}

Bohren, C. F. 1987. Multiple scattering of light and some of its observable consequences. Am. F. Phys., 55(6), 524-533.

Bohren, C. F. and B. R. Barkstrom. 1974. Theory of the optical properties of snow. F. Geophys. Res., 79 (30), 4527-4535.

Born, M. and E. Wolf. 1980. Principles of optics. Sixth edition. New York, etc., Pergamon Press.

Debye, P., H. R. Anderson and H. Brumberger. 1957. Scattering by an inhomogeneous solid II. The correlation function and its application. 7 . Appl. Phys., 28(6), 679-683.

De Haan, J. F., P. B. Bosma and J.W. Hovenier. 1987. The adding method for multiple scattering calculations of polarized light. Astron. Astrophys., 183, 371-391.

Ishimaru, A. 1978. Wave propagation and scattering in random media. New York, Academic Press.

Marshall, S. E. 1989. A physical parameterization of snow albedo for use in climate models. (Ph.D. thesis, National Center for Atmospheric Research, Boulder, CO.) (NCAR Cooperative Thesis 123.)

Mätzler, C. 1987. Applications of the interaction of microwaves with the natural snow cover. Remote Sensing Rev., 2(2), 259-387.

Mätzler, C. 1997. Autocorrelation functions of granular media with free arrangement of spheres, spherical shells or ellipsoids. F. Appl. Phys., 81 (3), 1509-1517.

Mätzler, C. 1998a. Improved born approximation for scattering in a granular medium. F. Appl. Phys., 83(11), 6111-6117.

Mätzler, C. 1998b. Microwave properties of ice and snow. In Schmitt, B, C. De Bergh and M. Festou, eds. Solar system ices. Dordrecht, etc., Kluwer Academic Publishers, 241-257. (Astrophysics and Space Science Library 227.)

Meador, W. E. and W. R. Weaver. 1980. Two-stream approximations to radiative transfer in planetary atmospheres: a unified description of existing methods and a new improvement. 7. Atmos. Sci., 37, 630-643.

Salisbury, J.W., D. M. D'Aria and A. Wald. 1994. Measurements of thermal infrared spectral reflectance of frost, snow, and ice. 7. Geophys. Res., 99(B12), 24,235-24,240.

Sergent, C., E. Pougatch, M. Sudul and B. Bourdelles. 1993. Experimental investigation of optical snow properties. Ann. Glaciol., 17, 281-287.

Sergent, C., G. Leroux, E. Pougatch and F. Guirado. 1998. Hemisphericaldirectional reflectance measurements of natural snows in the 0.9 $1.45 \mu \mathrm{m}$ spectral range: comparison with adding-doubling modelling. Ann. Glaciol., 26, 59-63.

Wald, A. E. 1994. Modeling thermal infrared $(2-14 \mu \mathrm{m})$ reflectance spectra of frost and snow. 7. Geophys. Res., 99 (B12), 24,241-24,250.

Warren, S. G. 1984. Optical constants of ice from the ultraviolet to the microwave. Appl. Opt., 23(8), 1206-1225.

Wiesmann, A. and C. Mätzler. 1999. Microwave emission model of layered snowpacks. Remote Sensing of Environment, 70 (3), 307-316.

Wiesmann, A., C. Mätzler and T. Weise. 1998. Radiometric and structural measurements of snow samples. Radio Sci., 33(2), 273-289.

Wiscombe, W. J. and S. G. Warren. 1980. A model for the spectral albedo of snow. I. Pure snow. 7. Atmos. Sci., 37(12), 2712-2733. 